\title{
Pathology of Peripheral Neuroblastic Tumors
}

\author{
Hiroyuki Shimada ${ }^{1}$, Hideki Sano ${ }^{2}$ and Florette K. Hazard ${ }^{1}$ \\ ${ }^{1}$ Department of Pathology and Pediatrics, Stanford University School of Medicine, Stanford, CA, USA, \\ ${ }^{2}$ Department of Pathology Oncology, Fukushima Medical University Hospital, Fukushima, Japan
}

Peripheral neuroblastic tumors (pNTs including Neuroblastoma, Ganglioneuroblastoma and Ganglioneuroma) are biologically and clinically heterogeneous. In order to develop efficient clinical treatment protocols of this disease, patients are stratified into different risk groups based on the combination of so-called prognostic factors. In this review, the prognostic factors are classified as clinically defined (Clinical staging and Age at diagnosis), histopathologically defined [International Neuroblastoma Pathology Classification (INPC) distinguishing Favorable Histology (FH) Group and Unfavorable Histology (UH) Group] and genetically/molecularly defined [MYCN oncogene amplification, DNA index, Segmental chromosomal aberrations, ALK (Anaplastic Lymphoma Kinase) mutation/amplification and Abnormal maintenance/elongation of telomeres], are outlined. According to the Children's Oncology Group (COG) Neuroblastoma studies, the survival rate of patients in low- or intermediate-risk group is more than $90 \%$. In contrast, the survival rate of patients in the high-risk group remains less than $50 \%$ despite currently available high-intensity and multimodal therapy. As described in the genetically/molecularly defined prognostic factors, tumors in the high-risk group, majority of which are classified into the UH group according to the INPC, are molecularly heterogeneous. Based on the recent progress of precision medicine along with our correlative analyses between molecular alterations and their morphological manifestations, four subgroups; i.e., MYC subgroup, TERT subgroup, ALT subgroup and Null subgroup, are identified immunohistochemically in the $\mathrm{UH}$ group for future management of the patients with this unique disease.

Key Words: Peripheral neuroblastic tumors, International Neuroblastoma Pathology Classification, Prognostic factors, Precision medicine, MYC-family protein, Telomere abnormalities
pISSN 2233-5250 / elSSN 2233-4580 https://doi.org/10.15264/cpho.2020.27.2.73 Clin Pediatr Hematol Oncol 2020;27:73 86

Received on September 24, 2020

Revised on October 8, 2020

Accepted on October 8, 2020

\author{
Corresponding Author: \\ Hiroyuki Shimada \\ Department of Pathology and \\ Pediatrics, Stanford University \\ School of Medicine, 300 Pasteur \\ Drive, Stanford, CA 94305, USA \\ Tel: +1-650-498-2098 \\ Fax: +1-650-736-1473 \\ E-mail: hshimada@stanford.edu \\ ORCID ID: orcid.org/0000-0001-5025-7507
}

\section{Prologue}

Tumors arising from the sympathoadrenal lineage of neural crest-derived tissues are collectively known as peripheral neuroblastic tumors (pNTs) and include Neuroblastoma, Ganglioneuroblastoma and Ganglioneuroma. pNTs are the third most common childhood neoplasm after leukemias and brain tumors, the most common ne- oplasms during the first year of life, and the most common extra-cranial solid tumors in the first 2 years of life. pNTs are composed of biologically different groups and demonstrate a wide spectrum of clinical courses to include spontaneous regression, tumor maturation, and aggressive progression refractory to therapy. These clinical behaviors are closely associated with defects in various molecular mechanisms that regulate sympathetic nervous system development [1]. Recent advances in tumor biol- 
ogy and normal embryology have increased understanding of the functions of the molecular markers and enhanced efforts to find actionable targets for precision medicine.

\section{Prognostic Factors}

Historically, various prognostic factors predicting clinical behavior of pNTs are reported. Those are clinically, histopathologically, and genetically/molecularly defined factors. In order to develop efficient clinical management protocols, risk-grouping systems have been established to stratify affected patients by combinations of the risk factors (please see Risk grouping section). These important prognostic factors are presented below.

\section{1) Clinically defined factors}

Clinical stage and Age at diagnosis are well known prognostic factors. Historically, they have been accepted and included in the risk grouping systems.

\section{(1) Clinical stage}

There are two widely used clinical staging systems: They are INSS (International Neuroblastoma Staging System) [2] and INRGSS (International Neuroblastoma Risk Group Staging System) [3]. The INSS is based on the post-surgical evaluation of disease extent, and distinguishes localized disease (Stage 1, 2 and 3), metastatic disease (Stage 4) and special metastatic disease with favorable clinical outcome (Stage 4S). The INRGSS is based on pre-surgical determination of disease extent, and localized disease is distinguished into two Stages based on presence or absence of Image-Defined Risk Factors (IDRFs): they are L1 (localized disease without IDRFs) and L2 (localized disease with IDRFs). INRGSS also include Stage M (metastatic disease) and Stage MS (special metastatic disease). By definition, disease status of Stage $4 \mathrm{~S}$ is the same as that of MS, but age at diagnosis of the former is less than one year (365 days) and the latter is less than 1.5 years (548 days). More than $50 \%$ of pNT patients have metastatic disease at the time of diagnosis. It is reported that the event-free survival (EFS) rate and overall survival (OS) rate for the patients with stage $4 / \mathrm{M}$ disease are $35 \%$ and $42 \%$, respectively. In contrast those for the patients with localized and special metastatic disease are $83 \%$ and $91 \%$, respectively [4].

\section{(2) Age at diagnosis}

In pNTs, the patient's age at diagnosis has been used as one of the prognostic indicators. Historically, one year of age was used as the cut-off for distinguishing a better prognostic group ( $<365$ days) from a worse prognostic group ( $\geq 365$ days). However, in 2005, London et al. reported that (1) the prognostic contribution of age to the clinical outcome is continuous in nature: Whichever age cut-off was adopted, survival rate of younger patients was always better than that of older patients, and (2) there was statistical evidence of an age cut-off greater than one year for risk stratification [5]. Since then, national and international clinical studies have been moving the age cut-off for prognostic distinction from one year (365 days) to 18 months (548 days). Age should be regarded as a surrogate for other risk factors. For example, the International Neuroblastoma Pathology Classification (INPC, see below) already has a built-in age cutoff of 18 months for distinguishing prognostic groups. Furthermore, Sano et al. demonstrated that the INPC was able to add independent prognostic information beyond the contribution of age [6]. In other words, the INPC clearly distinguishes two prognostic groups (Favorable Histology Group and Unfavorable Histology Group, the former identifying significantly better prognosis than the latter) in various age groups, such as $<$ vs. $\geq 12$ months (365 days), $<$ vs. $\geq 18$ months (548 days), and $<$ vs. $\geq 24$ months (730 days) of age on diagnosis (Fig. 1).

\section{2) Histopathologically defined factors}

Histopathology has also been included in the risk grouping systems. It is interesting to note that pNTs can offer one of the best models to investigate biologically significant relationship between genomic/molecular alterations and morphologic manifestations (please see Subgrouping of Unfavorable Histology Neuroblastoma and Precision Medicine section).

\section{(1) Categories and subtypes (Fig. 2)}

"Neuroblastoma" is often used as an omnibus term for all tumors in the pNTs. In 1999, the International Neuro- 

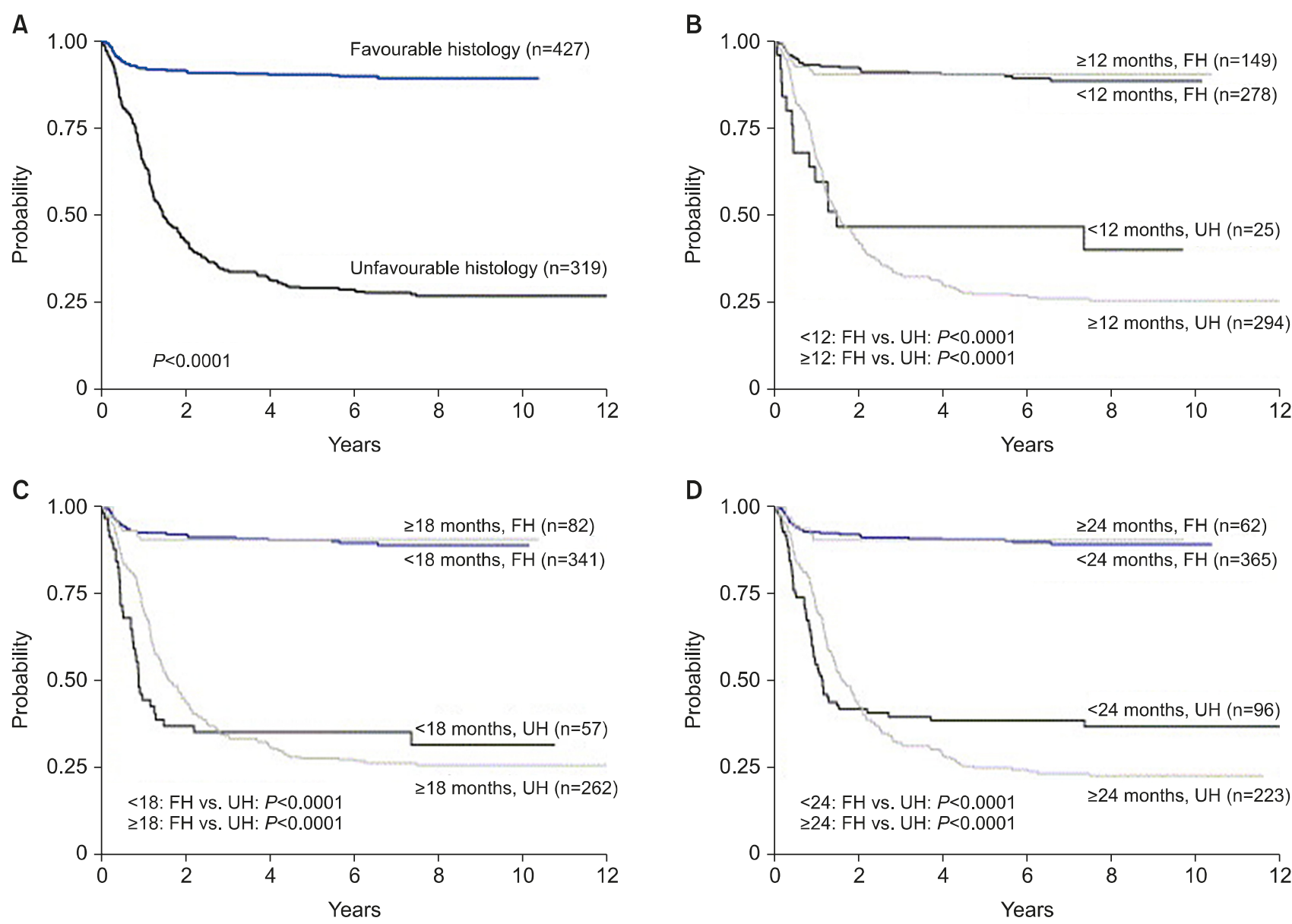

Fig. 1. Event-free survival curves according to the International Neuroblastoma Pathology Classification. The Classification clearly distinguishes prognostic groups for all patients (A), for patient groups over and under 12 months of age (B), for patient groups over and under 18 months of age (C), and for patient groups over and under 20 months of age (D). Note: This Figure is made by combining a portion of Figure 2 and Figure 3 in Sano et al. (2006) International neuroblastoma pathology classification adds independent prognostic information beyond the prognostic contribution of age. Eur J Cancer 2006; 42:1113-1119 [6].

blastoma Pathology Committee defined 4 categories [7]: They are Neuroblastoma (Schwannian stroma-poor; no or limited Schwannian stromal development occupying up to $50 \%$ of tumor tissue; Fig. $2 \mathrm{~A}-\mathrm{C}$ ), Ganglioneuroblastoma, Intermixed (Schwannian stroma-rich; Schwannian stromal development occupying $>50 \%$ of tumor tissue; Fig. 2D), Ganglioneuroma (Schwannian stroma-dominant; predominantly Schwannian stroma containing individual or clusters of ganglion; Fig. 2E) and Ganglioneuroblastoma, Nodular (composite, Schwannian stroma-rich/stromadominant and stroma-poor; Fig 2E). The first three categories represent tumor maturation prompted by "crosstalk" between neuronal tumor cells and Schwannian stromal cells supported by various signaling pathways, in- cluding trkA/NGF signaling and $\mathrm{Nrg} 1 / \mathrm{ErbB}$ signaling [8-10]. As described by Willis [11], pNTs are embryonal tumors: It is believed that all Ganglioneuromas were once Neuroblastomas in their early stage of tumor development. The fourth category of Ganglioneuroblastoma, nodular is defined as composite or multi-clonal tumor. Among all the pNTs reviewed and filed at the COG Neuroblastoma Pathology Reference Laboratory, approximately $80 \%$ are in the Neuroblastoma category and the rests are in the Ganglioneuroblastoma, Intermixed (9\%), Ganglioneuroma (3\%), and Ganglioneuroblastoma, Nodular (8\%) category (unpublished data).

In the Neuroblastoma category, there are three subtypes depending on the grade of neuroblastic differ- 

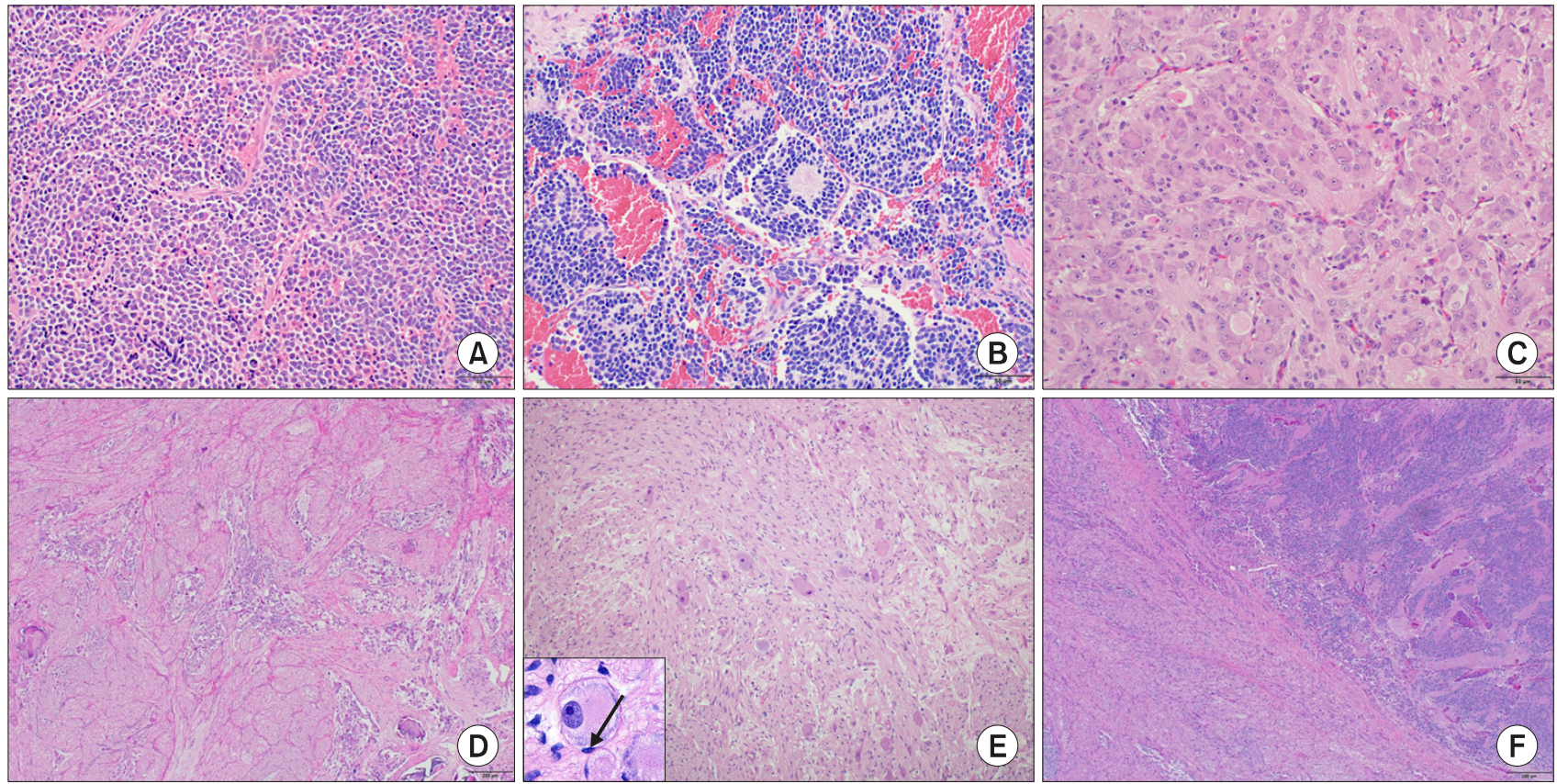

Fig. 2. Histologic categories and subtypes in peripheral neuroblastic tumors. Undifferentiated subtype (A), poorly differentiated subtype (B) and differentiating subtype (C) of Neuroblastoma (Schwannian stroma-poor) category; Ganglioneuroblastoma, Intermixed (Schwannian stroma-rich) category (D); Ganglioneuroma (Schwanninan stroma-dominant) category (E, inset: a ganglion cell covered with a satellite cell indicated by arrow) and Ganglioneuroblastoma, Nodular (composite, Schwannnian stroma-rich and stroma-poor) category [F, Ganglioneuroblastoma, Intermixed component (lower left) and Neuroblastoma component (upper right) of this example].

entiation: They are Undifferentiated (totally undifferentiated tumor without potential of neuroblastic differentiation, immunohistochemical and/or molecular tests required for confirming the diagnosis; $3 \%$ in the Neuroblastoma category; Fig. 2A), Poorly differentiated (tumor with or without potential of further neuroblastic differentiation, diagnosis confirmed by identifying neurite formation by tumor cells; $89 \%$ in the Neuroblastoma category; Fig. 2B), and Differentiating (tumor with more than $5 \%$ of neoplastic cells having an appearance of differentiating neuroblast; $8 \%$ in the Neuroblastoma category; Fig. 2C) subtype. In the tumor tissue of the Ganglioneuroblastoma, Intermixed (one step behind of complete maturation towards Ganglioneuroma), neuritic processes produced by the neuroblastic cells are not completely incorporated in the cytoplasm of Schwann cells, so that foci of naked neurites are still identified microscopically (Fig. 2D). While in the tumor tissue of the Ganglioneuroma (final stage of tumor maturation), all neuritic processes produced by ganglion cells are imme- diately incorporated in the cytoplasm of Schwann cells, so that no naked neuritic processes are detected (Fig. 2E). In the Ganglioneuroma category, two subtypes, maturing and mature, are recorded: Ganglion cells are embedded in the Schwannian stroma of both subtypes, and the latter is composed only of completely mature ganglion cells covered with satellite cells (Fig. 2E, inset).

(2) International Neuroblastoma Pathology Classification (INPC)

The INPC was established in 1999 by adopting the original Shimada classification [12] with minor modifications [13] and revised in 2003 [14]. According to the INPC, pNTs are classified into either Favorable Histology (FH) Group or Unfavorable Histology (UH) Group (Please see Fig. 1A). As shown in Table 1, all tumors in the Ganglioneuroblastoma, Intermixed category and in the Ganglioneuroma category are FH with an excellent clinical outcome. While tumors in the Neuroblastoma category and Ganglioneuroblastoma, Nodular (composite tumor of Ganglioneuroblastoma, Intermixed or Ganglioneuroma 
Table 1. International Neuroblastoma Pathology Classification $[7,13]$

\begin{tabular}{|c|c|c|}
\hline & Favorable histology & Unfavorable histology \\
\hline \multicolumn{3}{|l|}{ Neuroblastoma (Schwannian stroma-poor) } \\
\hline Undifferentiated subtype & & All Tumors (Any MKI, Any Age) \\
\hline Poorly differentiated subtype & Low or Intermediate MKI, $<548$ days & $\begin{array}{l}\text { Low or Intermediate MKI, } \geq 548 \text { days } \\
\text { High MKI, Any Age }\end{array}$ \\
\hline Differentiating subtype & Low MKI, $<5$ years & $\begin{array}{l}\text { Any } \mathrm{MKI}, \geq 5 \text { years } \\
\text { Intermediate } \mathrm{MKI}, \geq 548 \text { days } \\
\text { High } \mathrm{MKI}, \text { Any Age }\end{array}$ \\
\hline $\begin{array}{l}\text { Ganglioneuroblastoma, intermixed } \\
\text { (Schwannian stroma-rich) }\end{array}$ & \multicolumn{2}{|l|}{ All Tumors } \\
\hline \multicolumn{3}{|c|}{ Ganglioneuroma (Schwannian stroma-dominant) } \\
\hline Maturing subtype & \multicolumn{2}{|l|}{ All Tumors } \\
\hline Mature subtype & \multicolumn{2}{|l|}{ All Tumors } \\
\hline $\begin{array}{l}\text { Ganglioneuroblastoma, Nodular } \\
\text { (composite, Schwannian stroma-rich/ } \\
\text { Stroma-dominant and stroma-poor) }\end{array}$ & \multicolumn{2}{|c|}{$\begin{array}{l}\text { Favorable histology vs. unfavorable histology distinction: based on age-linked } \\
\text { evaluation of Grade of neuroblastic differentiation (subtype) and MKI of the neuro- } \\
\text { blastoma nodule }\end{array}$} \\
\hline
\end{tabular}

MKI, Mitosis Karyorrhexis Index; Low, (<100/5,000 cells); Intermediate, (100-200/5,000 cells); High, (>200/5,000 cells).

containing neuroblastoma nodule) category are distinguished into the FH or UH Group based on age-dependent evaluation of histologic indicators of the tumor or neuroblastoma nodule: They are the grade of neuroblastic differentiation (i.e., subtype of Undifferentiated, Poorly differentiated or Differentiating; see Categories and Subtypes above) and the mitotic and karyorrhectic activities [MKI, Mitosis-Karyorrhexis Index; low $(<100 /$ 5,000 cells), intermediate (100-200/5,000 cells) or high (>200/5,000 cells)]. Determination of MKI class is based on averaging the activities by counting mitotic and karyorrhectic cells in multiple representative microscopic fields. In other words, MKI class is not determined by counting the activities from the hottest, i.e. most condensed, fields. Cells with mitosis and nuclear fragmentations (i.e. karyorrhexis) are included in the counting, while simply hyperchromatic (darkly stained) nuclei are excluded.

FH tumors fit into an age-appropriate framework and display differentiation/maturation from Poorly differentiated subtype to Differentiating subtype of Neuroblastoma, then to Ganglioneuroblastoma, Intermixed and finally to Ganglioneuroma. In order to observe tumor differentiation/maturation, however, a certain period of time, i.e., in vivo latent period, seems to be required in each phase. The INPC defines two critical time points: "up to
18 months" from Neuroblastoma Poorly differentiated subtype to Neuroblastoma Differentiating subtype, and "up to 60 months" to Ganglioneuroblastoma, Intermixed or Ganglioneuroma. In other words, even though the tumors in the FH Group have a potential of differentiation/maturation, they should follow the age-appropriate framework. Accordingly, (1) patients $<18$ months ( $<548$ days) of age at diagnosis with Neuroblastoma, Poorly differentiated subtype, (2) patients $<5$ years $(<60$ months) at diagnosis with Neuroblastoma, Differentiating subtype, and (3) all Ganglioneuroblastoma, Intermixed and Ganglioneuroma (usually diagnosed in older children and young adults) fall into the FH Group with an excellent prognosis $[15,16]$. The prognostic implications of MKI are also age-dependent: (1) Low MKI tumors in the patients $<60$ months of age on diagnosis, and (2) Intermediate MKI tumors in the patients $<18$ months of age on diagnosis come within the framework of the $\mathrm{FH}$ Group [17].

UH Group includes tumors of the (1) Neuroblastoma, Undifferentiated subtype in any age-group, tumors of the Neuroblastoma, (2) Poorly differentiated subtype in patients over 18 months of age, and (3) tumors of the Neuroblastoma, Differentiating subtype in patients over 60 months of age. Those tumors are considered to have limited or no differentiation/maturation potential and 
they are outside the age-appropriate framework. As for MKI classes, (1) High MKI NB tumors in any age group, (2) Intermediate MKI tumors $\geq 18$ months of age at diagnosis, and (3) Low MKI tumors $\geq 60$ months of age at diagnosis are also outside the framework. A significant correlation between MYCN amplification and High MKI has been reported [18,19]. Again, those tumors with morphology indicators outside the frame of FH Group are assigned to the UH Group.

According to the INPC, survival rate of patients in the FH group is estimated to be approximately $90 \%$, whereas that of the UH group has remained approximately 50\% for years [6]. In summary, clinically unfavorable behaviors, such as treatment-resistance and aggressive progression, are observed in patients having $\mathrm{UH}$ tumors in the Neuroblastoma category and UH neuroblastoma nodule in the Ganglioneuroblastoma, Nodular category. It should be noted that the INPC is applicable only to the tumor specimens obtained before starting chemotherapy/irradiation therapy [7,13]. Post-chemotherapy morphologic changes representing "acute chemotherapy effects" do not reliably predict clinical outcome. Based on our experience, those changes, such as neuroblastic differentiation and even focal Schwannian stromal development, observed in UH tumors after chemotherapy are often misleading. Large areas of necrosis and extensive hemosiderin deposition are frequently seen in the $\mathrm{UH}$ tumors but are rarely found in post-therapy FH tumors. We should, however, conduct further study on recurrent tumors after chemo/irradiation therapy, since they could demonstrate different genetic/molecular characteristics from those detected at the time of initial diagnosis due possibly to clonal selection/evolution or therapy-induced events during the individual clinical courses.

\section{3) Genetically/molecularly defined factors}

Genomic/molecular alterations closely associated with clinically unfavorable tumor behaviors are listed in this portion.

\section{(1) MYCN oncogene amplification}

Amplification of the $M Y C N$ oncogene, located on chromosome 2 p 24.3 , is seen in approximately $20-25 \%$ of
Neuroblastoma tumors and considered as a major oncogenic driver. A large region including the $M Y C N$ locus becomes amplified and detected as extra-chromosomal double minutes (DMs), and may be randomly integrated into any chromosome as homogenously staining regions (HSRs). By fluorescence in situ hybridization (FISH) using a double staining method, $M Y C N$ status is determined as amplified (increased signals four folds or more than reference signals of chromosome 2; Fig. 3, inset), gain (increased signals but less than four folds of reference signals of chromosome 2), or non-amplified [20]. In recent years, the importance of heterogenous $M Y C N$ intra-tumoral amplification associated with a diverse aggressive phenotype has emerged [21]. MYCN-amplified tumors are associated with advanced stage and aggressive clinical behavior [22,23]. They demonstrate characteristic histology of either undifferentiated or poorly differentiated subtype with a high MKI and are classified into the UH Group according to the INPC (Fig. 3) [18,19].

\section{(2) DNA index}

Determination of DNA index by flow cytometric analysis has been used for predicting clinical outcome of patients with Neuroblastoma tumors. Tumors presenting with numeric whole-chromosomal copy number gains without structural abnormalities (hyperdiploid, DNA in-

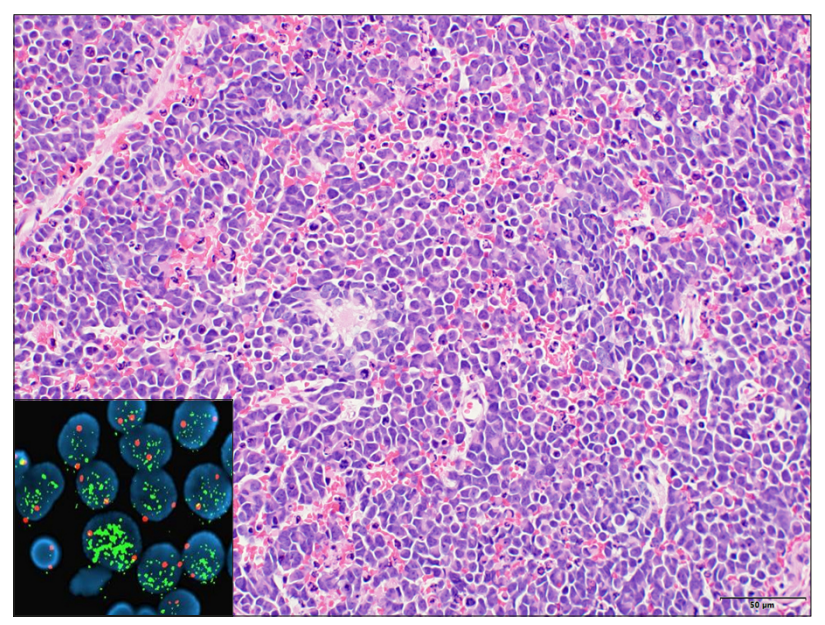

Fig. 3. MYCN-amplified neuroblastoma with a high MitosisKaryorrhexis Index. [Inset: MYCN amplification detected by fluorescence in situ hybridization - double staining method (green signals of numerous double minutes containing MYCN oncogene and red signals of Chromosome 2 centromere)]. 
dex $>1)$ are associated with an excellent prognosis. In contrast, diploid tumors (DNA index=1) are associated with a poor clinical outcome [24,25]. The prognostic effect of ploidy pattern is lost in children over the age of two years, possibly because "DNA index $>1$ " tumors in older children typically have a number of structural abnormalities.

\section{(3) Segmental chromosome aberrations}

Various segmental chromosomal aberrations have been reported as factors predictive of poor prognosis of [2629]. Chromosome $1 \mathrm{pLOH}$ (loss of heterozygosity), reported in $25-35 \%$ of cases, is often associated with MYCN amplification. Most deletions are located distal to 1p36, where possible tumor suppressor genes or genes controlling differentiation of neuroblasts could be located. Chromosome $11 \mathrm{qLOH}$, identified in $35-45 \%$ of cases, is often associated with older patients, and rarely occurs in combination with $M Y C N$ amplification. Among 11q aberrations, $11 \mathrm{q} 23$ is the most common site for deletion. $17 \mathrm{q}$ trisomy, detected in approximately $60 \%$ of cases, may be the most prevalent genetic abnormality in this disease. 17q gain can occur independently, but it is frequently found as part of an unbalanced translocation between chromosomes 1 and 17. The 17q breakpoints vary, but a region has been defined at 17q21-qter.

The presence of any of these chromosomal alterations (1p deletion, 11q deletion, and 17q gain) was reported to add prognostic information to the age and stage of neuroblastoma patients without $M Y C N$ amplification [30]. 6q loss is also reported in a subgroup of extremely aggressive neuroblastoma [31]. Recently age-dependency of the prognostic impact of genomic aberrations in localized resectable $M Y C N$ non-amplified neuroblastomas is reported [32].

(4) ALK (anaplastic lymphoma kinase) mutation/amplification

ALK is a receptor tyrosine kinase, expressed in the developing sympathoadrenal lineage of the neural crest. Historically, $A L K$ mutations were reported in hereditary neuroblastoma cases [33]. $A L K$-activating mutations and amplification are also found in approximately $10 \%$ of sporadic neuroblastomas [34,35]. ALK abnormalities (mutations and amplification) resulting in its protein over- expression seem to be responsible for its oncogenic activity [36] by causing dysregulation of multiple signaling transduction pathways and allowing the uncontrolled proliferation of neuroblasts. In addition, $A L K$ gene amplification and F1174 mutations, which are among the most active forms of mutations in in-vitro assays, are associated with $M Y C N$ amplification [37,38]. Recently clinical trials with $A L K$ inhibitors have been started for patients with $A L K$ mutated/amplified neuroblastoma. It is interesting to note that autophagy has been observed in neuroblastoma treated with ALK inhibitors, opening a new challenge for neuroblastoma therapy [39,40]. However, the prognostic significance of $A L K$ overexpression itself still remains controversial [41]. In this regard, Regairaz et al. reported that expression of ALK protein and its active form of pALK was immunohistochemically detected in many neuroblastomas independent of $A L K$ mutations/amplification [42].

\section{(5) Abnormal maintenance/elongation of telomeres}

Two mechanisms associated with telomere abnormality are reported in this disease: They are TERT (telomerase reverse transcriptase) overexpression and ALT (alternative lengthening of telomeres) phenotype. TERT is the protein component of telomerase, and higher levels of expression are observed in both MYC-driven neuroblastoma (neuroblastoma overexpressing either $\mathrm{n}-\mathrm{MYCN}$ protein or c-MYC protein: please see MYC subtype in Subgrouping of Unfavorable Histology Neuroblastomas and Precision Medicine below) and non-MYC-driven neuroblastoma. In the former case, TERT is the direct transcriptional target of MYC family proteins [43]. In the latter case, it is likely a result of long-range genomic rearrangements of the TERT gene. TERT promoter hypermethylation may be also involved in its activating mechanism [44-47]. High telomerase activity is reported to be predictive of reduced patient survival.

Most tumors with ATRX loss due to ATRX (alpha-thalassemia/mental retardation syndrome X-linked) gene mutation show the ALT phenotype [48] found in children over 5 years of age with a poor clinical outcome [49]. ATRX mutation is rarely observed in the MYC-driven neuroblastoma, and is not associated with TERT over- 
expression. ALT phenotype is also reported in rare tumors with DAXX (Death Domain Associate Protein) loss [50].

\section{Risk Grouping}

Since pNTs are biologically/clinically heterogeneous, patients with this disease are divided into different risk groups. There are 2 major risk-grouping systems: One is the COG Risk Classification System (Currently in the process of revision) and the other is the INRG (International Neuroblastoma Risk Group) (Table 2). Both systems use a combination of various prognostic factors, such as clinical stage, age at diagnosis, histopathology and molecular/genomic properties. Historically, the COG Risk Classification System (distinguishing low-, intermediate-, and high-risk group) has contributed to actual patient stratification and protocol assignment [51,52]. In contrast, the primary purpose of the INRG (distinguishing very low-, low-, intermediate-, and high-risk) is to facili- tate the comparison of risk-based clinical trials conducted in different regions and countries [4]. By using the COG Risk Classification, approximately $90 \%$ of survival rate is expected by surgery alone for the patients in the low-risk group, and a similar survival rate is also expected by biopsy plus non-aggressive chemotherapy for children in the intermediate-risk group. In contrast, the survival rate of the patients in the high-risk group remains approximately $50 \%$ despite currently available high-intensity multimodal therapy.

\section{Subgrouping of Unfavorable Histology Neuroblastoma and Precision Medicine}

It is noted that most of the tumors in the high-risk group are UH with unfavorable clinical and genomic/ molecular factors. Those high-risk or UH tumors are also heterogeneous and associated with different molecular backgrounds (see Genetically/molecularly defined factors

Table 2. International Neuroblastoma Risk Group (INRG) consensus pretreatment classification schema [4]

\begin{tabular}{|c|c|c|c|c|c|c|c|c|}
\hline $\begin{array}{l}\text { INRG } \\
\text { Stage }\end{array}$ & $\begin{array}{c}\text { Age } \\
\text { (month) }\end{array}$ & Histologic category & $\begin{array}{l}\text { Grade of tumor } \\
\text { differentiation }\end{array}$ & MYCN & $\begin{array}{c}11 q \\
\text { Aberration }\end{array}$ & Ploidy & \multicolumn{2}{|c|}{$\begin{array}{l}\text { Pretreatment } \\
\text { risk group }\end{array}$} \\
\hline $\mathrm{L} 1 / \mathrm{L} 2$ & & GN maturing; GNB intermixed & & & & & A & Very low \\
\hline \multirow[t]{2}{*}{ L1 } & & Any, except GN maturing or & & NA & & & $\mathrm{B}$ & Very low \\
\hline & & GNB intermixed & & Amp & & & $\mathrm{K}$ & High \\
\hline \multirow[t]{6}{*}{ L2 } & $<18$ & Any, except GN maturing or & & NA & No & & $\mathrm{D}$ & Low \\
\hline & & GNB intermixed & & & Yes & & G & Intermediate \\
\hline & $\geq 18$ & GNB nodular; & Differentiating & NA & No & & $\mathrm{E}$ & Low \\
\hline & & neuroblastoma & & & Yes & & $\mathrm{H}$ & Intermediate \\
\hline & & & Poorly differentiated & NA & & & & \\
\hline & & & or undifferentiated & Amp & & & $\mathrm{N}$ & High \\
\hline \multirow[t]{5}{*}{ M } & $<18$ & & & NA & & Hyperdiploid & $\mathrm{F}$ & Low \\
\hline & $<12$ & & & NA & & Diploid & 1 & Intermediate \\
\hline & 12 to $<18$ & & & NA & & Diploid & $J$ & Intermediate \\
\hline & $<18$ & & & Amp & & & $\mathrm{O}$ & High \\
\hline & $\geq 18$ & & & & & & $\mathrm{P}$ & High \\
\hline \multirow[t]{3}{*}{ MS } & $<18$ & & & $\mathrm{NA}$ & No & & $\mathrm{C}$ & Very low \\
\hline & & & & & Yes & & $\mathrm{Q}$ & High \\
\hline & & & & Amp & & & $\mathrm{R}$ & High \\
\hline
\end{tabular}

Pretreatment risk group $\mathrm{H}$ has two entries. 12 months=365 days; 18 months=547 days; blank field= "any"; diploid (DNA index 1.0); hyperdiploid (DNA index $>1.0$ and includes near-triploid and near-tetraploid tumors); very low risk (5-year EFS $>85 \%$ ); low risk (5-year EFS $>75 \%$ to $\leq 85 \%$ ); intermediate risk (5-year EFS $\geq 50 \%$ to $\leq 75 \%$ ); high risk (5-year EFS $<50 \%$ ). GN, ganglioneuroma; GNB, ganglioneuroblastoma; Amp, amplified; NA, not amplified; L1, localized tumor confined to one body compartment and with absence of image-defined risk factors (IDRFs); L2, locoregional tumor with presence of one or more IDRFs; M, distant metastatic disease (except stage MS); MS, metastatic disease confined to skin, liver and/or bone marrow in children $<18$ months of age; EFS, event-free survival. Note: This table is the same as Fig. 2 in Cohn SL et al. (2008) The International Neuroblastoma Risk Group (INRG) Classification System: An INRG Task Force Report / Clin Oncol (2008) 27:289-297 [4]. 
above). Based on the fact that neuroblastoma can offer one of the best models for investigating biologically significant relationship between molecular alterations and their morphologic characteristics, Ikegaki et al. recently proposed four subgroups in UH neuroblastomas for precision medicine (Fig. 4). They are MYC subgroup (overexpressing MYCN or MYC protein), TERT subgroup (TERT overexpression due to genomic abnormalities), ALT subgroup (Alternative Lengthening of Telomere due to ATRX loss) and Null subgroup. These subgroups are immunohistochemically identified by their protein makers that could be potential therapeutic targets [53].

\section{1) MYC subgroup}

Approximately $90 \%$ of $M Y C N$-amplified tumors overexpress n-MYC protein [54]. Recent studies show that the protein overexpression rather than the gene amplification is critical for predicting aggressive tumor behavior [55]. Also found are tumors with augmented expression of c-MYC protein in this disease. Apparently, n-MYC/ c-MYC-MAX protein heterodimer formations activate downstream molecular targets by binding to EnhancerBox (E-box) sequences, which leads to aggressive tumor growth. In fact, patients with neuroblastomas expressing

\begin{tabular}{|c|c|c|c|c|c|c|}
\hline & \multirow[b]{2}{*}{ Subgroups } & \multicolumn{3}{|c|}{ Immunohistochemistry (IHC) markers } & \multirow[b]{2}{*}{ Histologic markers } & \multirow{2}{*}{$\begin{array}{l}\text { Predicted } \\
\text { survival based } \\
\text { on current } \\
\text { therapy }\end{array}$} \\
\hline & & $\begin{array}{c}\text { Pan-MYC } \\
\text { or (MYCN/MYC) }\end{array}$ & TERT & ATRX & & \\
\hline $\begin{array}{c}\text { MYC-driven } \\
\text { neuroblastomas }\end{array}$ & $\begin{array}{c}\text { MYC } \\
(\mathrm{MYCN} / \mathrm{MYC})\end{array}$ & Overexpression & $\begin{array}{l}\text { Overexpression } \\
\text { or No over- } \\
\text { expression }\end{array}$ & Retention & $\begin{array}{l}\text { Nucleolar hypertrophy } \\
\text { (including large cell } \\
\text { neuroblastoma) }\end{array}$ & Dismal \\
\hline \multirow{3}{*}{$\begin{array}{l}\text { Non-MYC-driven } \\
\text { neuroblastomas }\end{array}$} & TERT & $\begin{array}{l}\text { No over- } \\
\text { expression }\end{array}$ & Overexpression & Retention & $\begin{array}{l}\text { Salt-and pepper nuclei } \\
\text { conventional } \\
\text { neuroblastoma }\end{array}$ & Dismal \\
\hline & ALT & $\begin{array}{l}\text { No over- } \\
\text { expression }\end{array}$ & $\begin{array}{l}\text { No over- } \\
\text { expression }\end{array}$ & Loss & $\begin{array}{l}\text { Salt-and pepper nuclei } \\
\text { conventional } \\
\text { neuroblastoma }\end{array}$ & Dismal \\
\hline & Null & $\begin{array}{l}\text { No over- } \\
\text { expression }\end{array}$ & $\begin{array}{l}\text { No over- } \\
\text { expression }\end{array}$ & Retention & $\begin{array}{l}\text { Salt-and pepper nuclei } \\
\text { conventional } \\
\text { neuroblastoma }\end{array}$ & $\begin{array}{l}\text { Better } \\
\text { response }\end{array}$ \\
\hline
\end{tabular}

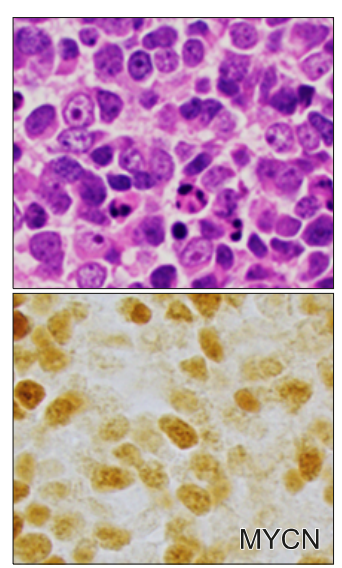

MYCN overexpression

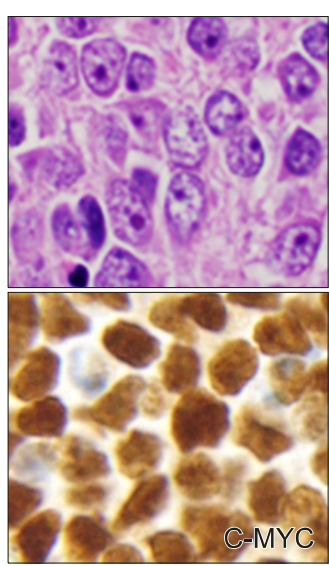

MYC overexpression large cell NB

MYC subgroup

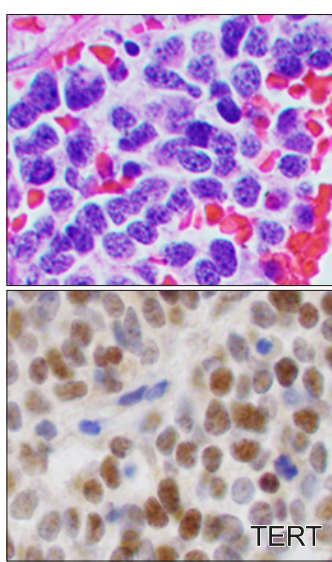

TERT overexpression

TERT subgroup

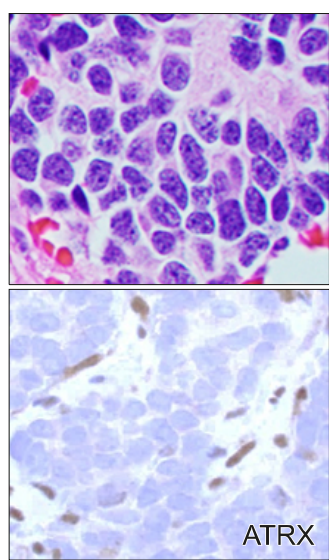

ATRX loss (retained by the endothelial cells)

ALT subgroup

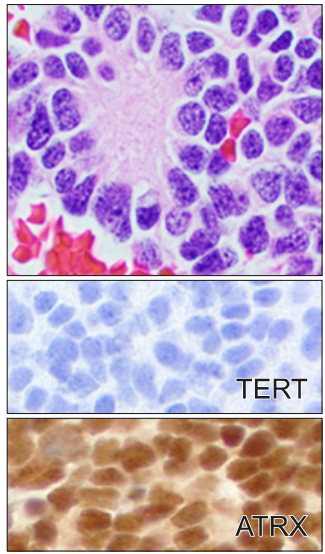

MYCN/MYC (-)
TERT $(-)$

ATRX (+)

Null subgroup

Fig. 4. Four subgroups in Unfavorable Histology Neuroblastoma. TERT, telomerase reverse transcriptase; ATRX, alpha-thalassemia/ mental retardation syndrome X-linked; ALT, alternative lengthening of telomeres. Note: This Figure is made by combining Table 1 and Fig. A1 in Ikegaki et al. (2019) Subgrouping of Unfavorable Histology Neuroblastomas with Immunohistochemistry toward Precision Prognosis and Therapy Stratification. JCO Precis Oncol 3:10 [53]. 
immunohistochemically detectable and higher levels of MYC-family protein (n-MYC and c-MYC) have a dismal clinical outcome. It is reported that tumors overexpressing n-MYC protein comprised approximately 20\% of neuroblastomas in the undifferentiated/poorly differentiated subtypes, and 3-year EFS of the patients was $46.2 \pm 12.0 \%$. While tumors overexpressing c-MYC protein were found in approximately $10 \%$ of tumors in the same subtypes, and 3-year EFS of the patients was $43.4 \pm$ 23.1\% (Fig. 5) [56]. Therefore we have defined these neuroblastomas as "MYC family-driven neuroblastomas" and they belong to UH according to INPC.

It is noted that augmented expression of the MYCfamily protein is characteristically associated with prominent nucleolar formation (nucleolar hypertrophy) that is considered as a putative site of MYCN/MYC RNA synthesis and accumulation (Fig. 6A-C) [54-58]. Due to the presence of prominent nucleoli, indicative of hyperactive rRNA synthesis and protein translation, MYC-driven neuroblastomas are morphologically distinguishable from the conventional neuroblastomas with so-called "saltand-pepper" nuclei. In contrast to n-MYC overexpressing neuroblastoma, it is extremely rare to see the gene amplification in c-MYC overexpressing neuroblastoma
[59]. In this regard, other molecular mechanisms for high c-MYC protein expression independent of the genomic amplification, such as the oncogene activation through enhancer hijacking by translocations, focal enhancer amplification near to the $\mathrm{c}-M Y C$-coding region (8q24) or MK2-mediated OCT4 transcriptional activation of the c-MYC gene, have been reported [60,61].

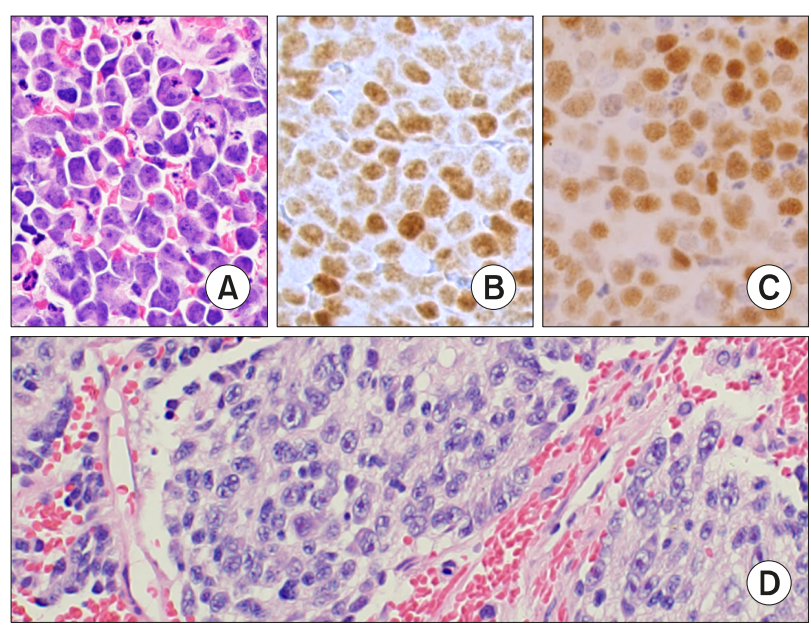

Fig. 6. MYC-driven neuroblastoma. Tumor demonstrating characteristic prominent nucleolar formation (nucleolar hypertrophy) (A), tumor overexpressing $n-M Y C$ protein ( $B$, immunohistochemistry), tumor overexpressing C-MYC protein (C, immunohistochemistry) and large cell neuroblastoma (D).
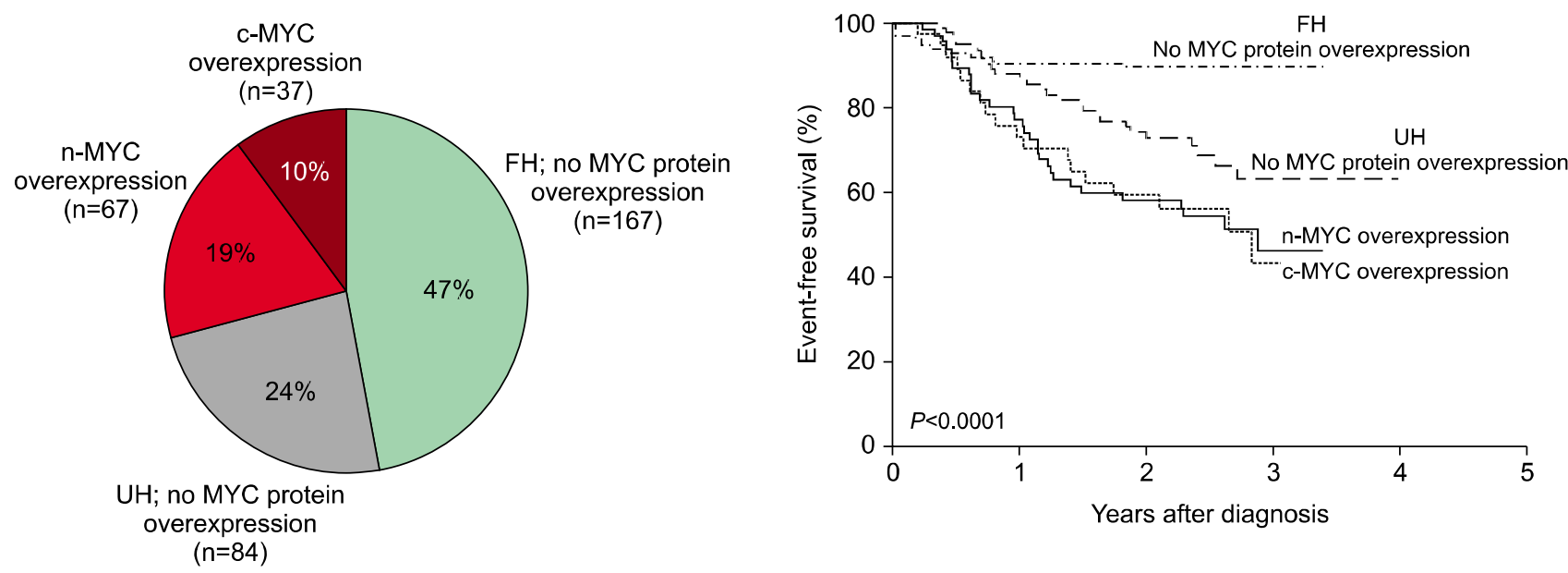

Total 355 cases (20 UD \& 335 PD)

Fig. 5. MYC-family protein (n-MYC or c-MYC) expression in Neuroblastoma, undifferentiated and poorly differentiated subtype. FH, favorable histology; UH, unfavorable histology; UD, undifferentiated subtype; PD, poorly differentiated subtype. Event-free survival of MYC-driven neuroblastoma is significantly lower than that of non-MYC-driven neuroblastomas. Note: This figure is from Figure 3 in Wang LL, et al. (2015) Augmented expression of MYC and/or MYCN protein defines highly aggressive MYC-driven neuroblastoma: a Children's Oncology Group study. Br J Cancer 113:57-63 [57]. 
The histologically defined, rare and very aggressive "large cell neuroblastoma (LCN)" is also included in this group (Figs. 4C, 6D) [62]. This is an extreme form of the MYC-driven neuroblastoma that demonstrates large cell appearance with bull's eye-like enlarged vesicular nuclei containing one to few very prominent nucleoli supporting exceedingly higher levels of either MYCN or MYC protein expression. Their "euchromatin-rich" open nuclei suggest stemness of the tumor cells [63]. Though the nuclear morphology of LCN cells is often similar to that of differentiating neuroblasts, these two cell types should be strictly distinguished. The tumor cells in LCN have larger nuclei than conventional neuroblasts and their $\mathrm{cy}^{-}$ toplasm is similarly scant. In contrast, differentiating neuroblasts have abundant cytoplasm with a diameter of at least two times larger than that of the nucleus [64].

It is a highly formidable task to directly target the MYC-family proteins with small molecules [65]. Hence, many investigators have sought indirect approaches to down regulate MYC-family protein expression in tumor cells. The strategies currently under consideration include, but not limited to, transcriptional repression of MYCN/MYC genes by BET-bromodomain inhibitors [66, 67] and CDK (Cyclin-dependent kinase) inhibitors [68,69] or destabilization of MYCN by Aurora kinase A inhibitors [70], etc. We also reported that small molecules, such as CX-5461 (a potent RNA Pol I inhibitor) and Halofuginone (a potent protein translation inhibitor), could effectively down-regulate MYC-family protein expression at the level of hypertrophic nucleoli of the neuroblastoma cells by interfering rRNA synthesis and protein translation [33].

\section{2) TERT subgroup}

As mentioned above, telomere elongation by increased TERT (telomere reverse transcriptase) activity can be detected immunohistochemistry in both MYC-driven and non-MYC-driven neuroblastomas in any age group [71]. In this subgroup, non-MYC-driven tumors having TERT rearrangements or promoter hypermethylation $[44,47,72]$ are included. Tumor cells in this subgroup seem to have salt-and-pepper nuclei without nucleolar hypertrophy.

In the treatment of TERT overexpressing neuroblastoma, telomerase inhibitors can be considered. Imetelstat (GRN163L) [73] is a potent and specific inhibitor of telomerase by binding with high affinity to TERC (telomerase RNA component). Interestingly, sorafenib has been shown to synergize with Imetelstat to inhibit growth of mouse xenografts of human cancer [74]. Sorafenib is a FDA-approved kinase inhibitor [75], and its synergistic effect with imetelstat appears to be due to the p21 attenuating activity [74].

\section{3) ALT subgroup}

Most tumors with $A T R X$ mutations leading to loss of ATRX expression show the ALT phenotype [49], and the protein loss can easily be detected by immunohistochemistry. Tumors in the MYC subgroup and TERT subgroup do not develop the ALT phenotype. Tumor cells in this subgroup, like those in the TERT subgroup, have salt-and-pepper nuclei without nucleolar hypertrophy.

Since ATRX loss is due to structural alterations in ATRX gene [76], it would be difficult to regain the protein expression in neuroblastoma. If so, is there any other way by which the ALT phenotype can be suppressed? To address this question, we need to understand the mechanism of how ATRX loss leads to the ALT phenotype. It has become evident that the acquisition of ALT phenotype utilizes the DNA replication stress response [77], which involves a cascade of events, including the obligatory activation of ATR (Ataxia Telangiectasia and Rad3 related) kinase. AZD6738 is a novel potent and selective inhibitor of ATR kinase with IC 50 values of less than $1 \mu \mathrm{M}$ in cell-based assays [78] and it would effectively target ALT tumor cells.

4) Null subgroup

Neuroblastomas without MYC-family protein overexpression, TERT protein overexpression and ATRX protein loss are tentatively placed into the Null subgroup. Without having any other known specific molecular abnormalities, we hypothesize that tumors in this subgroup may respond to treatment according to the current highrisk protocols.

Mutually exclusive relationships likely exist among tu- 
mors in the MYC subgroup, TERT subgroup and ALT subgroup. These subgroups constitute the vast majority of therapy-resistant and UH tumors of this disease. Thus, targeted therapies against MYC-family overexpression, TERT overexpression and ALT phenotype should improve outcome of patients with the high-risk neuroblastomas.

\section{Epilogue}

Towards the end of this review, we would like to express our cordial thanks to the fellow clinicians and researchers. In order to make any meaningful progress in this field of pediatric oncology, we really need a great deal of support/collaboration from our colleagues nationally and internationally. With a long history of the cooperative studies, we have identified prognostic factors clinically, histopathologically and genetically/molecularly, and keep improving risk grouping system. However, the survival rate of the patients with high-risk neuroblastoma, many of them are $\mathrm{UH}$ according to the INPC, still remains very low at the "far-from" satisfactory level. Now we are moving to a new stage of precision "pathway targeting" medicine and creating subgroups in the Unfavorable Histology Group by immunohistochemically identifying protein markers that are associated with aggressive clinical behaviors through different molecular mechanisms.

\section{Conflict of Interest Statement}

The authors have no conflict of interest to declare.

\section{References}

1. Huber K, Janoueix-Lerosey I, Kummer W, Rohrer H, Tischler AS. The sympathetic nervous system: malignancy, disease, and novel functions. Cell Tissue Res 2018;372:163-17.

2. Brodeur GM, Pritchard J, Berthold F, et al. Revisions of the international criteria for neuroblastoma diagnosis, staging, and response to treatment. J Clin Oncol 1993;11:1466-77.

3. Monclair T, Brodeur GM, Ambros PF, et al. The International Neuroblastoma Risk Group (INRG) staging system: an INRG Task Force report. J Clin Oncol 2009;27:298-303.

4. Cohn SL, Pearson AD, London WB, et al. The International
Neuroblastoma Risk Group (INRG) classification system: an INRG Task Force report. J Clin Oncol 2009;27:289-97.

5. London WB, Castleberry RP, Matthay KK, et al. Evidence for age cutoff greater than 365 days for neuroblastoma risk group stratification in the Children's Oncology Group. J Clin Oncol 2005;23:6459-65.

6. Sano H, Bonadio J, Gerbing RB, et al. International neuroblastoma pathology classification adds independent prognostic information beyond the prognostic contribution of age. Eur J Cancer 2006;42:1113-9.

7. Shimada H, Ambros I, Dehner PL, Hata J, Joshi VV, Roald B. Terminology and morphologic criteria of neuroblastic tumors: recommendation by the International Neuroblastoma Pathology Committee. Cancer 1999;86:349-63.

8. Liu S, Tian Y, Chlenski A, et al. Cross-talk between Schwann cells and neuroblasts influences the biology of neuroblastoma xenografts. Am J Pathol 2005;166:891-900.

9. Brodeur GM, Minturn JE, Ho R, et al. Trk receptor expression and inhibition in neuroblastomas. Clin Cancer Res 2009;15: 3244-50.

10. Pajtler KW, Mahlow E. Odersky A, et al. Neuroblastoma in dialog with its stroma: NTRK1 is a regulator of cellular crosstalk with Schwann cells. Oncotarget 2014;5:11180-92.

11. Willis RA. The borderland of embryology and pathology. 2nd ed. London: Butterworth \& Co. Ltd, 1962.

12. Shimada H, Chatten J, Newton WA Jr, et al. Histopathologic prognostic factors in neuroblastic tumors: definitions of subtypes of ganglioneuroblastoma and an age-linked classification of neuroblastoma. J Nat Cancer Institute 1984;73: 405-16.

13. Shimada H, Ambros IA, Dehner LP, et al. The International Neuroblastoma Pathology Classification (the Shimada System). Cancer 1999;86:364-72.

14. Peuchmaur M, d'Amore ESG, Joshi VV, et al. Revision of the International Neuroblastoma Pathology Classification: confirmation of favorable and unfavorable prognostic subsets in ganglioneuroblastoma, nodular. Cancer 2003;98:2274-81.

15. De Bernardi B, Gambini C, Haupt R, et al. Retrospective study of childhood ganglioneuroma. J Clin Oncol 2008;26:1710-6.

16. Okamatsu C, London WB, Naranjo A, et al. Clinicopathological characteristics of ganglioneuroma and ganglioneuroblastoma: a report from the CCG and COG. Ped Blood Cancer 2009;53:563-9.

17. Teshiba R, Kawano S, Wang LL, et al. Age-dependent prognostic effect by Mitosis-Karyorrhexis Index (MKI) in neuroblastoma: a report from the Children's Oncology Group. Pediatr Dev Pathol 2014;17:441-9.

18. Shimada H, Stram D, Chatten J, et al. Identification of subsets of neuroblastomas combined histopathologic and N-myc analysis. J Nat'l Cancer Inst 1995;87:1470-6.

19. Goto S, Umehara S, Gerbing RB, et al. Histopathology and MYCN status in peripheral neuroblastic tumors: a report from the Children's Cancer Group. Cancer 2001;92:2699-708.

20. Ambros PF, Ambros IM, Brodeur GM, et al. International consensus for neuroblastoma molecular diagnostics: report from the International Neuroblastoma Risk Group (INRG) Biology Committee. Br J Cancer 2009;100:1471-82. 
21. Berbegall AP, Villamón E, Piqueras M, et al. Comparative genetic study of intratumoral heterogenous MYCN amplified neuroblastoma versus aggressive genetic profile neuroblastic tumors. Oncogene 2016;35:1423-32.

22. Brodeur GM, Seeger RC, Schwab M, Varmus HE, Bishop JM. Amplification of $\mathrm{N}$-myc in untreated human neuroblastomas correlates with advanced disease stage. Science 1984;224:11214.

23. Seeger RC, Brodeur GM, Sather H, et al. Association of multiple copies of the N-myc oncogene with rapid progression of neuroblastomas. N Engl J Med 1985;313:1111-6.

24. Look AT, Hayes FA, Nitschke R, McWilliams NB, Green AA. Cellular DNA content as a predictor of response to chemotherapy in infants with unresectable neuroblastoma. N Engl J Med 1984;311:231-5.

25. George RE, London WB, Cohn SL, et al. Hyperdiploid plus nonamplified MYCN confers a favorable prognosis in children 12 to 18 months old with disseminated neuroblastoma: a Pediatric Oncology Group study. J Clin Oncol 2005;23: 6466-73.

26. Schleiermacher G, Delattre O, Peter M, et al. Clinical relevance of loss heterozygosity of the short arm of chromosome 1 in neuroblastoma: a single institution study. Int J Cancer 1996;69:73-8.

27. Attiyeh EF, London WB, Mosse YP, et al. Chromosome 1p and 11q deletions and outcome in neuroblastoma. N Engl J Med 2005;353:2243-53.

28. Caren H, Kryh H, Nethander M, et al. High-risk neuroblastoma tumors with $11 \mathrm{q}$-deletion display a poor prognostic, chromosome instability phenotype with later onset. Proc Natl Acad Sci U S A 2010;107:4323-8.

29. Bown N, Cotterill S, Lastowska M, et al. Gain of chromosome arm 17q and adverse outcome in patients with neuroblastoma. N Engl J Med 1999;340:1954-61.

30. Schleiermacher G, Nosseri V, London WB, et al. Segmental chromosomal alterations have prognostic impact in neuroblastoma: a report from the INRG project. Br J Cancer 2012; 107:1418-22

31. Depuydt P, Boeva V, Hocking TD, et al. Genomic amplifications and distal 6q loss: novel markers for poor survival in highrisk neuroblastoma patients. J Natl Cancer Inst 2018;110: 1084-93.

32. Ambros IM, Tonini GP, Potschger U, et al. Age-dependency of the prognostic impact of tumor genomics in localized resectable MYCN non-amplified neuroblastomas. Report From the SIOPEN Biology Group on the LNESG Trials and a COG Validation Group. J Clin Oncol 2020;JCO1802132.

33. Mossé YP, Laudenslager M, Longo L, et al. Identification of ALK as a major familial neuroblastoma predisposition gene. Nature 2008;455:930-5.

34. Chmielecki J, Bailey M, He J, et al. Genomic profiling of a large set of diverse pediatric cancers identifies known and novel mutations across tumor spectra. Cancer Res 2017;77: 509-19.

35. Bellini A, Bernard V, Leroy Q, et al. Deep sequencing reveals occurrence of subclonal ALK mutations in neuroblastoma at diagnosis. Clin Cancer Res 2015;21:4913-21.
36. Mosse YP. Anaplastic lymphoma kinase as a cancer target in pediatric malignancies. Clin Cancer Res 2015;22:546-52.

37. Umapathy G, El Wakil A, Witek B, et al. The kinase ALK stimulates the kinase ERK5 to promote the expression of the oncogene MYCN in neuroblastoma. Sci Signal 2014;7:ra102.

38. De Brouwer S, De Preter K, Kumps C, et al. Meta-analysis of neuroblastomas reveals a skewed ALK mutation spectrum in tumors with MYCN amplification. Clin Cancer Res 2010; 16:4353-62.

39. Aveic S, Pantile M, Seydel A, et al. Combating autophagy is a strategy to increase cytotoxic effects of novel ALK inhibitor entrectinib in neuroblastoma cells. Oncotarget 2016;7:564663.

40. Aveic S, Tonini GP. Resistance to receptor tyrosine kinase inhibitors in solid tumors: can we improve the cancer fighting strategy by blocking autophagy? Cancer Cell Int 2016;16:62.

41. Passoni L, Longo L, Collini $\mathrm{P}$, et al. Mutation-independent anaplastic lymphoma kinase overexpression in poor prognosis neuroblastoma patients. Cancer Res 2009;69:7338-46.

42. Regairaz M, Munier F, Sartelet H, et al. Mutation-independent activation of the anaplastic lymphoma kinase in neuroblastoma. Am J Pathol 2016;186:435-45.

43. Koh CM, Khattar E, Leow SC, et al. Telomerase regulates MYC-driven oncogenesis independent of its reverse transcriptase activity. J Clin Invest 2015;125:2109-22.

44. Lindner S, Bachmann HS, Odersky A, et al. Absence of telomerase reverse transcriptase promoter mutations in neuroblastoma. Biomed Rep 2015;3:443-6.

45. Lindsey JC, Schwalbe EC, Potluri S, et al. TERT promoter mutation and aberrant hypermethylation are associated with elevated expression in medulloblastoma and characterize the majority of non-infant SHH subgroup tumours. Acta Neuropathol 2014;127:307-9.

46. Lee S, Borah S, Bahrami A. Detection of aberrant TERT promoter methylation by combined bisulfite restriction enzyme analysis for cancer diagnosis. J Mol Diagn 2017;19:378-86.

47. Valentijn LJ, Koster J, Zwijnenburg DA, et al. TERT rearrangements are frequent in neuroblastoma and identify aggressive tumors. Nat Genet 2015;47:1411-4.

48. Roake CM, Artandi SE. DNA repair: telomere-lengthening mechanism revealed. Nature 2016;539:35-6.

49. Cheung NK, Zhang J, Lu C, et al. Association of age at diagnosis and genetic mutations in patients with neuroblastoma. JAMA 2012;307:1062-71.

50. Kurihara S, Hiyama E, Onitake Y, Yamaoka E, Hiyama K. Clinical features of ATRX or DAXX mutated neuroblastoma. J Pediatr Surg 2014;49:1835-8.

51. Park JR, Bagatell R, London WB, et al. Children's Oncology Group's 2013 blueprint for research: neuroblastoma. Pediatr Blood Cancer 2013;60:985-93

52. Naranjo A, Irwin MS, Hogarty MD, Cohn SL, Park JR, London WB. Statistical Framework in Support of a Revised Children's Oncology Group Neuroblastoma Risk Classification System. JCO Clin Cancer Inform 2018;2:1-15.

53. Ikegaki N, Shimada H. Subgrouping of unfavorable histology neuroblastomas with immunohistochemistry toward precision prognosis and therapy stratification. JCO Precis Oncol 
2019;3:PO.18.00312.

54. Niemas-Teshiba R, Matsuno R, Wang LL, et al. MYC-family protein overexpression and prominent nucleolar formation represent prognostic indicators and potential therapeutic targets for aggressive high-MKI neuroblastomas: a report from the Children's Oncology Group. Oncotarget 2017;9;641632.

55. Suganuma R, Wang LL, Sano H, et al. Peripheral neuroblastic tumors with genotype phenotype discordance: a report from the Children's Oncology Group and the International Neuroblastoma Pathology Committee. Pediatr Blood Cancer 2013; 60:363-70.

56. Wang LL, Suganuma R, Ikegaki N, et al. Neuroblastoma of undifferentiated subtype, prognostic significance of prominent nucleolar formation, and MYC/MYCN protein expression: a report from the Children's Oncology Group. Cancer 2013;119:3718-26.

57. Wang LL, Teshiba R, Ikegaki N, et al. Augmented expression of MYC and/or MYCN protein defines highly aggressive MYC-driven neuroblastoma: a Children's Oncology Group study. Br J Cancer 2015;113:57-63.

58. Kobayashi C, Monforte-Munoz H, Gerbing RB, et al. Enlarged and prominent nucleoli may be indicative of MYCN amplification: a study of neuroblastoma (Schwannian stroma-poor), undifferentiated/poorly differentiated subtype with a high mitosis-karyorrhexis index. Cancer 2005;103:174-80.

59. Matsuno R, Gifford AJ, Fang J, et al. Rare MYC-amplified neuroblastoma with large cell histology. Pediatr Dev Pathol 2018;21:461-6.

60. Zimmerman MW, Liu Y, He S, et al. MYC drives a subset of high-risk pediatric neuroblastomas and is activated through mechanisms including enhancer hijacking and focal enhancer amplification. Cancer Discov 2018;8:320-35.

61. Wei SJ, Nguyen TH, Yang IH, et al. MYC transcription activation mediated by OCT4 as a mechanism of resistance to 13-cisRA-mediated differentiation in neuroblastoma. Cell Death Dis 2020;11:368.

62. Tornóczky T, Kálmán E, Kajtár PG, et al. Large cell neuroblastoma: a distinct phenotype with aggressive clinical behavior. New entity? Cancer 2004;100:390-7.

63. Ikegaki N, Shimada H, Fox AM, et al. Transient treatment with epigenetic modifiers yields stable neuroblastoma stem cells resembling aggressive large-cell neuroblastomas. Proc Natl Acad Sci U S A 2013;110:6097-102.

64. Tornóczky T, Semjén D, Shimada H, Ambros IM. Pathology of peripheral neuroblastic tumors: significance of prominent nucleoli in undifferentiated/poorly differentiated neuroblastoma. Pathol Oncol Res 2007;13:269-75.

65. Darnell JE Jr. Transcription factors as targets for cancer therapy. Nat Rev Cancer 2002;2:740-9.

66. Delmore JE, Issa GC, Lemieux ME, et al. BET bromodomain inhibition as a therapeutic strategy to target c-Myc. Cell 2011;146:904-17.

67. Puissant A, Frumm SM, Alexe G, et al. Targeting MYCN in neuroblastoma by BET bromodomain inhibition. Cancer Discovery 2013;3:308-23.

68. Christensen CL, Kwiatkowski N, Abraham BJ, et al. Targeting transcriptional addictions in small cell lung cancer with a covalent CDK7 inhibitor. Cancer Cell 2014;26:909-22.

69. Chipumuro E, Marco E, Christensen CL, et al. CDK7 inhibition suppresses super-enhancer-linked oncogenic transcription in MYCN-driven cancer. Cell 2014;159:1126-39.

70. Gustafson WC, Meyerowitz JG, Nekritz EA, et al. Drugging MYCN through an allosteric transition in Aurora kinase A. Cancer Cell 2014;26:414-27.

71. Nael A, Matsuno R, Shows J, et al. TERT overexpression in neuroblastoma. Society for Pediatric Pathology Fall Meeting Abstractbook 2017:4.

72. Peifer M, Hertwig F, Roels F, et al. Telomerase activation by genomic rearrangements in high-risk neuroblastoma. Nature 2005;526:700-4.

73. Dikmen ZG, Gellert GC, Jackson S, et al. In vivo inhibition of lung cancer by GRN163L: a novel human telomerase inhibitor. Cancer Res 2005;65:7866-73.

74. Gupta R, Dong Y, Solomon PD, et al. Synergistic tumor suppression by combined inhibition of telomerase and CDKN1A. Proc Natl Acad Sci U S A 2014;111:E3062-71.

75. Wilhelm S, Carter C, Lynch M, et al. Discovery and development of sorafenib: a multikinase inhibitor for treating cancer. Nat Rev Drug Discov 2006;5:835-44.

76. Cheung NK, Dyer MA. Neuroblastoma: developmental biology, cancer genomics and immunotherapy. Nat Rev Cancer 2013;13:397-411.

77. Flynn RL, Cox KE, Jeitany M, et al. Alternative lengthening of telomeres renders cancer cells hypersensitive to ATR inhibitors. Science 2015;347:273-7.

78. Vendetti FP, Lau A, Schamus S, Conrads TP, O’Connor MJ, Bakkenist CJ. The orally active and bioavailable ATR kinase inhibitor AZD6738 potentiates the anti-tumor effects of cisplatin to resolve ATM-deficient non-small cell lung cancer in vivo. Oncotarget 2015;6:44289-305. 